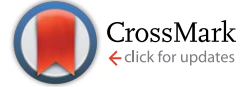

Cite this: RSC Adv., 2017, 7, 17593

Received 31st January 2017 Accepted 16th March 2017

DOI: 10.1039/c7ra01299c

rsc.li/rsc-advances

\section{Effect of melt and solution electrospinning on the formation and structure of poly(vinylidene fluoride) fibres $\dagger$}

\author{
Hanako Asai, ${ }^{*}$ Marina Kikuchi, Naoki Shimada and Koji Nakane
}

\section{Introduction}

Poly(vinylidene fluoride) (PVDF) is known as a piezoelectric polymer and is widely used in sensors, electroacoustic and electromechanical transducers, etc. ${ }^{\mathbf{1} 2}$ PVDF can form several types of crystal structures depending on the processing conditions. ${ }^{3}$ Among them, the $\beta$ crystalline phase, where the dipoles point in the same directions, shows remarkable piezoelectricity. ${ }^{1}$ In order to obtain the $\beta$ crystalline phase from a solution or melt states of PVDF, processes such as electrical poling and mechanical drawing are generally carried out. ${ }^{3}$

Recently, electrospinning techniques have been investigated to fabricate piezoelectric materials. Because electrospinning can prepare nanofibres by applying a high voltage to a polymer solution or a polymer melt, one can easily obtain piezoelectric textiles without post poling processes. For example, near-field electrospinning techniques enable in situ mechanical stretching and electrical poling and could produce piezoelectric PVDF nanofibres with high energy conversion efficiency. ${ }^{4,5}$ In addition, some researchers used a rotating collector to add mechanical drawing to the electrospun nanofibres. ${ }^{5-7}$ Considering that energy harvesting textiles have attracted attention in the field of wearable electronics, ${ }^{8}$ it is worth studying the relationship between spinning conditions and piezoelectricity.

There are many parameters for electrospinning, such as applied voltage, feeding rate, spinning solution concentration, and nozzle-to-collector distance. Recently, Shao et al. studied the effect of electrospinning parameters and polymer concentration on the crystal structure and the piezoelectric behaviour of the PVDF nanofibre mat. ${ }^{9}$ However, to the best of our knowledge, all

Frontier Fibre Technology and Science, Graduate School of Engineering, University of Fukui, 3-9-1 Bunkyo, Fukui, 910-8507, Japan.E-mail: h_asai@u-fukui.ac.jp

$\dagger$ Electronic supplementary information (ESI) available: Histograms for the diameters of M-ES and S-ES fibres. See DOI: 10.1039/c7ra01299c of the studied systems used solution electrospinning, and there has not been a report investigating the relationship between melt-electrospinning conditions and the piezoelectric properties. Here, as the relevant methods to melt-electrospinning, Lee et al. ${ }^{\mathbf{1 0}}$ and Hadimani et al. ${ }^{\mathbf{1 1}}$ reported an electric poling-assisted additive manufacturing (EPAM) process, and a continuous melt extrusion process with in-line poling, respectively. By using their processes, free-form shape or fibrous piezoelectric devices could be obtained. However, their processes could produce only sub-mm order filaments, and could not produce non-woven fabrics like electrospinning method. On the other hand, meltelectrospinning is one of the electrospinning techniques, where a polymer is melted or softened by heat and simultaneously spun by applying high electrostatic force, resulting in $\mu \mathrm{m}$-order fibres. Here, some researchers reported the piezoelectricity of PVDF was improved by high temperature, ${ }^{\mathbf{1 2 , 1 3}}$ while the others reported that the poling temperature was not as crucial parameter or high temperature was not proper. ${ }^{\mathbf{1 4 , 1 5}}$ Considering these researches, it is still not clear whether the high temperature used in melt-electrospinning is suitable for fabricating piezoelectric fibre mats or not.

Ogata et al. developed a new type of melt-electrospinning technique equipped with a $\mathrm{CO}_{2}$ laser melting device. ${ }^{16}$ In this technique, a rod like polymer sample is locally heated with a spot like laser beam, and fibres can be prepared. This laser melt-electrospinning does not spend much energy compared to conventional melt-electrospinning and does not use any organic solvent. However, because it used a rod-shaped polymer and a spot laser beam, it could fabricate only one fibre per onepoint laser irradiation, resulting in very low productivity. Then, Shimada et al. changed the laser shape from spot to linear and used sheet-formed polymer. ${ }^{17}$ By changing the laser and sample polymer shapes, they succeeded in making many Taylor corns, where the fibres were produced, and the productivity was significantly improved. 
In this study, we systematically investigated the effect of spinning parameters on the piezoelectric properties of PVDF fibre prepared by linear laser melt electrospinning (M-ES) and obtained fundamental knowledge about the relationship between the properties, structures, and spinning conditions. In addition, we compared the results with conventional solutionelectrospinning (S-ES).

\section{Experimental}

\subsection{Materials}

PVDF pellets were purchased from Sigma-Aldrich. The average molecular weight $\left(M_{\mathrm{w}}\right)$ of the PVDF was $\sim 180000 \mathrm{~kg} \mathrm{~mol}^{-1}$. $N, N$-Dimethylfolmamide (DMF) was purchased from Nacalai Tesque, inc. (Kyoto, Japan). All materials were used as received.

\subsection{Melt electrospinning using a linear laser device}

At first, PVDF pellets were heat-pressed at $190{ }^{\circ} \mathrm{C}$ with a $120 \mu \mathrm{m}$ spacer under about 4.9 MPa of pressure and cooled down slowly with keeping the pressure. Thus, we obtained a $c a .116 \mu \mathrm{m}$ thick PVDF sheet that was used for the following electrospinning. The schematic representation of the melt electrospinning device was shown in Fig. 1(a). The PVDF sheet was heated and melted by the laser beam, then drawn to the rotating collector by a drag force caused by a high voltage. The $\mathrm{CO}_{2}$ laser was generated from a spot like laser beam generator (Universal Laser System, ULR-50, wavelength $=10.6 \mu \mathrm{m}$ ). By using a custom-made optical system, we changed the shape of the laser beam from spot to linear form.

(a) M-ES

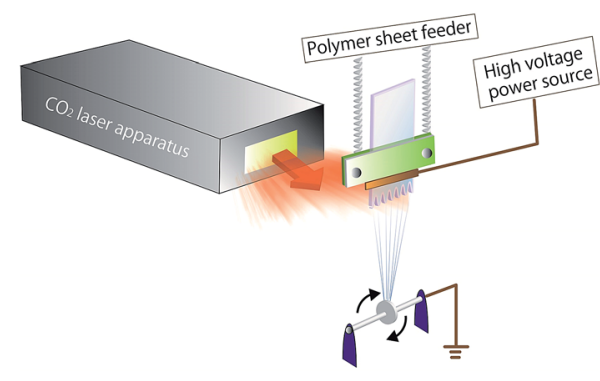

High speed rotating collector

(b) S-ES

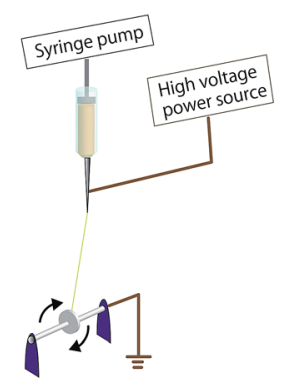

High speed rotating collector

Fig. 1 Schematic representation of (a) the melt electrospinning (M-ES) apparatus using a line-like laser melting system and (b) conventional solution electrospinning (S-ES) apparatus. The same rotating collector was used for both systems.
The details of the optical system are explained in our previous report. ${ }^{17}$ The feed rate of the polymer sheet was $1 \mathrm{~mm} \mathrm{~min}^{-1}$. The sheet-collector distance was $3.5 \mathrm{~cm}$. The diameter and width of the rotating collector were $7 \mathrm{~cm}$ and $7 \mathrm{~mm}$, respectively.

\subsection{Solution electrospinning}

The spinning solution was prepared by dissolving PVDF with DMF and acetone at $70{ }^{\circ} \mathrm{C}$. The fraction of the two solvents was $1: 1$ by weight. The solution concentration was fixed at $26 \mathrm{wt} \%$. Fig. 1(b) shows the S-ES apparatus used in this study. The same rotating collector as in the M-ES was used. The spinning nozzle was 21 gauge with a $0.51 \mathrm{~mm}$ inner diameter. The applied voltage was $-10 \mathrm{kV}$. All of the S-ES was carried out at a temperature and humidity ranging from $19{ }^{\circ} \mathrm{C}$ to $24{ }^{\circ} \mathrm{C}$, and $19 \%$ to $26 \%$, respectively. The feed rate was $0.6 \mathrm{~mL} \mathrm{~h}^{-1}$. The nozzle-collector distance was $3.5 \mathrm{~cm}$, which was also the same as the case of M-ES.

\subsection{Measurements}

The temperature of the PVDF sheet during the M-ES was monitored by using a thermographic camera (FSV-7000E, Apiste Corporation, Osaka, Japan) equipped with a custom-made filter to protect the apparatus from the laser beam.

Differential scanning calorimetry (DSC) curves were measured using a calorimeter (DSC-60, Shimadzu Corporation, Kyoto, Japan), at $10{ }^{\circ} \mathrm{C} \min ^{-1}$ from $30{ }^{\circ} \mathrm{C}$ to $250{ }^{\circ} \mathrm{C}$ in air.

Scanning electron microscopy (SEM) observations were carried out by using a Keyence scanning electron microscope (VE-9800, Keyence Co. Ltd., Osaka, Japan). The fibre samples were gold-sputter coated with an ion coater (SC-701, Sanyu Electron Co., Ltd., Tokyo, Japan). The average and the standard deviation of the fibre diameters were determined from 100 measurements using an image $\mathrm{J}$ software. Mat-form samples where many fibres were piled up were used for SEM observations as well as in all of the following experiments.

Attenuated total reflectance Fourier transform infrared spectroscopy (ATR-FTIR) data were obtained at room temperature using an IR spectrometer (IR Affinity-1, Shimadzu Corporation, Kyoto, Japan) equipped with a single reflection ATR accessory (MIRacle 10, Shimadzu Corporation, Kyoto, Japan) containing a diamond/ZnSe crystal.

$\mathrm{X}$-ray diffraction (XRD) measurements were carried out using an X-ray diffractometer (Ultima-IV, Rigaku Corporation, Tokyo, Japan), equipped with a sample holder for fibrous specimens. $\mathrm{Cu} \mathrm{K} \alpha$ radiation (wavelength, $1.54 \AA$ ) operated at $40 \mathrm{kV}$ and 20 $\mathrm{mA}$ was used, and the data were collected in the $2 \theta$ range from $10^{\circ}$ to $40^{\circ}$ at room temperature with the step angle of $0.05^{\circ}$.

Piezoelectric constant, $d_{33}$, values were measured by using piezometer system (PM300, Piezotest Pte. Ltd., Singapore) at $110 \mathrm{~Hz}$.

\section{Results and discussion}

\subsection{Condition of PVDF sheet during M-ES process}

Fig. 2 shows the thermographic image of the PVDF sheet during laser beam irradiation at $53 \mathrm{~W}$. The applied voltage was $-10 \mathrm{kV}$. 


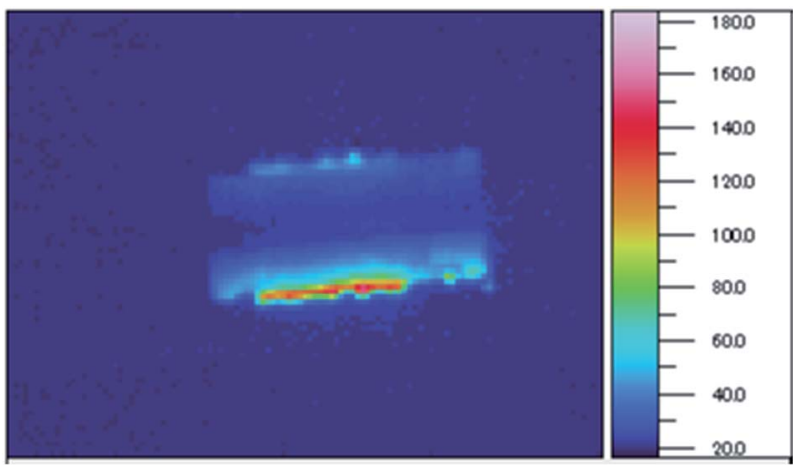

Fig. 2 Thermographic image of the PVDF sheet during laser beam irradiation of $\mathrm{M}$-ES at $53 \mathrm{~W}$. The applied voltage was $-10 \mathrm{kV}$. Temperature from $20{ }^{\circ} \mathrm{C}$ to $180{ }^{\circ} \mathrm{C}$ is represented by the colour difference.

The heated region of the PVDF sheet is indicated in red. The average maximum temperature of the sheet was $156.7 \pm 2.5^{\circ} \mathrm{C}$. Fig. 3 shows the results of DSC measurements of the PVDF sheet used for the M-ES. The melting point determined by DSC was $c a$. $170.5^{\circ} \mathrm{C}$. Therefore, it was found that the fibres prepared by $\mathrm{M}-$ ES were spun below the melting temperature by strong electrostatic force.

\subsection{Effect of the spinning condition on the fibre structure}

Fig. 4 summarises the SEM images of the PVDF fibre mats prepared by M-ES at different applied voltages and collector rotating speeds. The laser output power was fixed at $53 \mathrm{~W}$. Here, an electrospinning process was generally carried out at positive applied voltage, but we obtained only burnt fibres at positive voltage. Considering that PVDF is triboelectrically highly negative, ${ }^{18}$ it may be that the PVDF sheet was only slightly positively charged even at a positive applied voltage, and thus the electrostatic drawing force was not generated between the collector and the PVDF sheet, resulting in the overheating of the PVDF. Therefore, all of our experiments were carried out at negative applied voltage.

As the applied voltage was negatively increased, the orientation of the spun fibres became random, indicating that the insufficient neutralization of the charge of the fibres caused

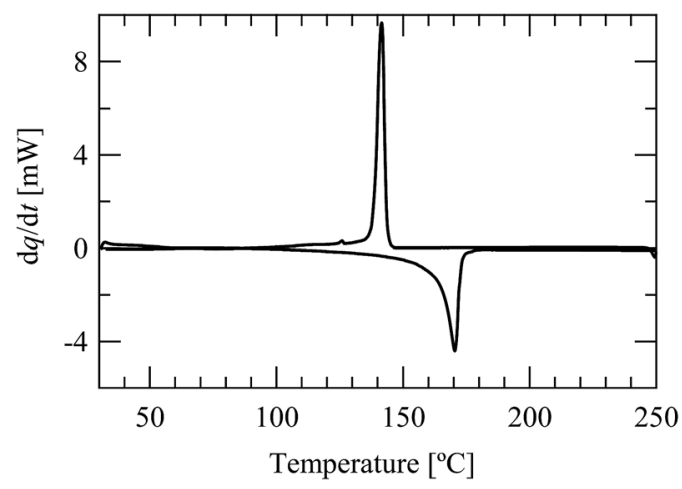

Fig. 3 DSC thermograms of the PVDF sheet used for M-ES.

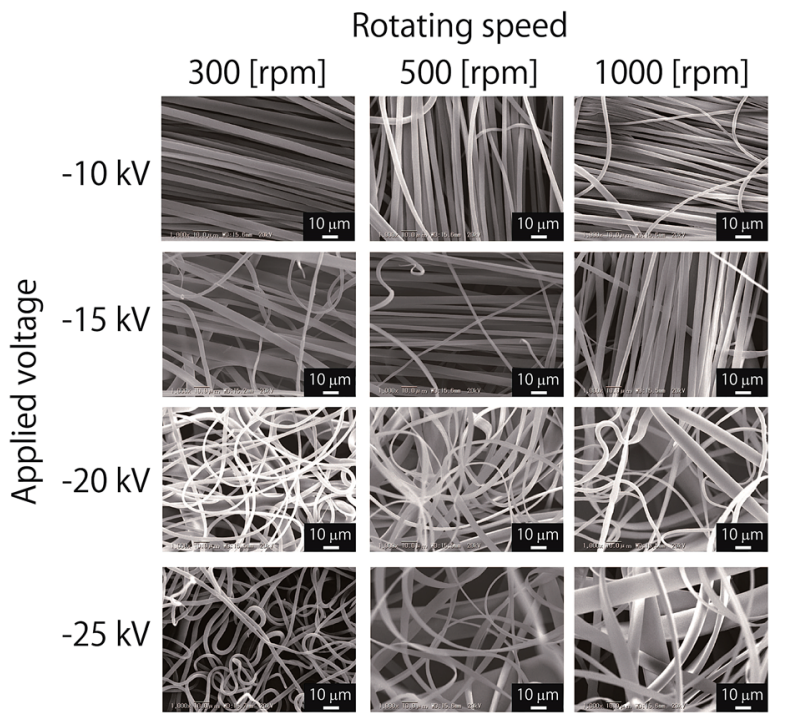

Fig. 4 SEM images of the fibre mats spun by M-ES at various applied voltages and rotating speeds. The laser output power and the polymer sheet feed rate were fixed at $53 \mathrm{~W}$ and $1 \mathrm{~mm} \mathrm{~min}^{-1}$, respectively.

electrostatic repulsion. In addition, too high applied voltage and rotating speed made fibres get drawn from the PVDF sheet before it was sufficiently melted, causing ribbon-like flat fibres $(-25$ kV, 1000 rpm, in Fig. 4).

The corresponding results of S-ES are shown in Fig. 5. As you can see, the diameter of the fibres was smaller than in the case of $\mathrm{M}$-ES, and the orientation of the fibres was achieved at higher rotating speeds. The fibre diameters obtained from the SEM photographs are summarised in Fig. 6. In the case of M-ES, the average diameter of fibres spun at $-10 \mathrm{kV}$ and $1000 \mathrm{rpm}$ was 4.2 $\pm 3.4 \mu \mathrm{m}$, while the S-ES fibre had an average diameter of $0.58 \pm$ $0.14 \mu \mathrm{m}$ under the same spinning conditions. The reason for the smaller diameters of S-ES fibres can be attributed to the different spinning mechanisms between these two spinning systems: in the case of M-ES, polymer sheet softened by the laser irradiation is drawn to the collector by electrostatic force, and fibres are obtained. On the other hand, in the case of S-ES, polymer solution is ejected from a spinning nozzle. The solvent of the ejected solution gradually evaporates during the spinning process, and only the remained polymer component forms fibres, resulting in the formation of finer fibres than the case of M-ES.

The fibre diameter of M-ES tended to increase with increasing rotating speed. This can also be attributed to the (a) 0 [rpm]

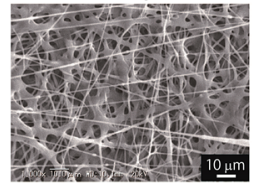

(b) 1000 [rpm]

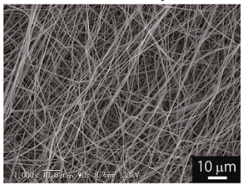

(c) 2000 [rpm]

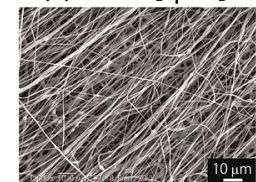

Fig. 5 SEM images of the nanofibre mats spun by S-ES at various rotating speeds: (a) $0 \mathrm{rpm}$, (b) $1000 \mathrm{rpm}$, and (c) $2000 \mathrm{rpm}$. The feed rate and the applied voltage were fixed at $0.6 \mathrm{~mL} \mathrm{~h}^{-1}$ and $-10 \mathrm{kV}$, respectively. 


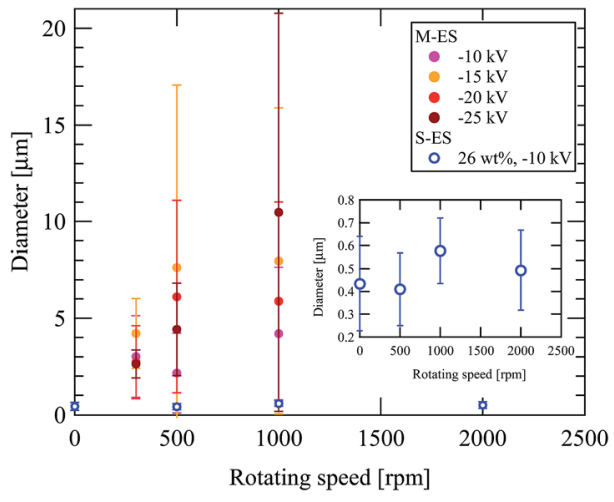

Fig. 6 Variation of the diameters of the M-ES and S-ES fibres as a function of applied voltage and rotating speed. The inset shows the magnified figure for S-ES fibres.

strong drawing caused by the higher rotating speed before sufficient heating of the sheet, and agrees with the thermography, and DSC results. The large errors of the M-ES fibres came from the wide distributions of the diameters as shown in the histograms (Fig. S1 $\dagger$ ). On the other hand, the fibre diameters of the S-ES fibres were not affected by the rotating speed, and its diameter distributions were narrower than the case of M-ES (Fig. S2 $\dagger$ ). This may be due to the stable spinning for S-ES.

\subsection{Effect of spinning conditions on the crystal structure}

Fig. 7 shows the XRD spectra for the (a) M-ES and (b) S-ES fibre mats spun at $500 \mathrm{rpm}$ and $-10 \mathrm{kV}$ after subtracting background data. According to the peak positions summarized by Jurczuk et al. ${ }^{19}$ we assigned the peak positions for $\alpha$ and $\beta$ phase, indicated by dashed lines in Fig. 7. As shown in these figures, the fibres spun by M-ES contained more $\alpha$ phase, while those spun by S-ES were rich in $\beta$ phase, though they showed small peaks assigned to $\alpha$ phases at $18.1^{\circ}, 26.6^{\circ}$ and $35.7^{\circ}$.

In addition, we compared the crystal structure of the fibres obtained from M-ES and S-ES. Fig. 8 shows the ATR-FTIR spectra

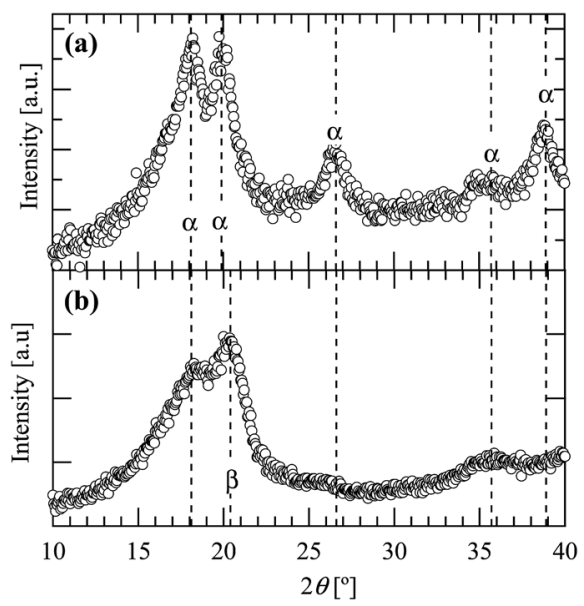

Fig. 7 XRD spectra for (a) M-ES and (b) S-ES (26 wt\%) fibre mats spun at $500 \mathrm{rpm}$, at $-10 \mathrm{kV}$.
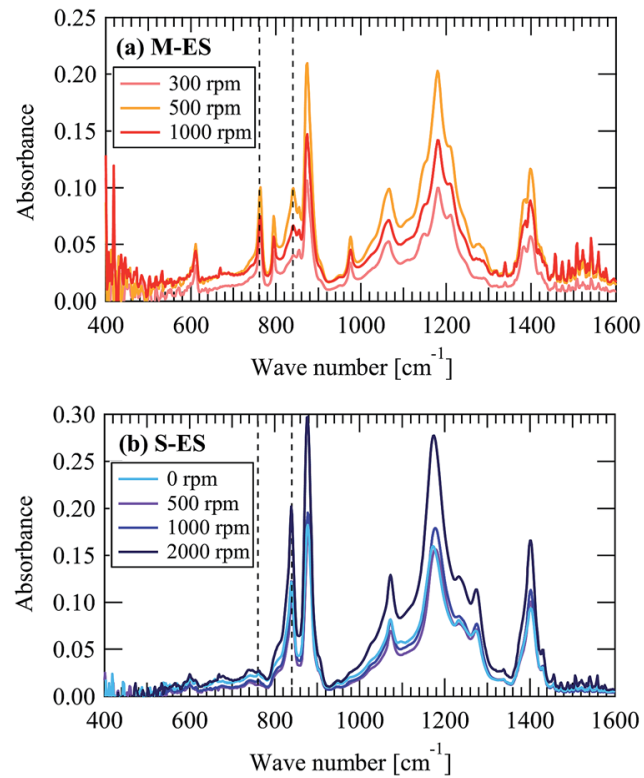

Fig. 8 ATR-FTIR spectra of the fibres spun by (a) M-ES and (b) S-ES with various rotating speeds. The applied voltage was $-10 \mathrm{kV}$. The broken lines indicate the positions of $761 \mathrm{~cm}^{-1}$ and $840 \mathrm{~cm}^{-1}$, respectively.

for (a) M-ES and (b) S-ES at various rotating speeds. The applied voltage was $-10 \mathrm{kV}$ for both spinning systems. In the reference, peaks at $761 \mathrm{~cm}^{-1}, 877 \mathrm{~cm}^{-1}$, and $976 \mathrm{~cm}^{-1}$ are assigned to $\alpha$ phase. ${ }^{9}$ They correspond to $\mathrm{CH}_{2}$ in-plane bending or rocking, $\mathrm{CH}_{2}$ out-of-plane bending or rocking, and $\mathrm{CH}_{2}$ twisting, respectively. ${ }^{9}$ Peaks at $840 \mathrm{~cm}^{-1}$ and $1274 \mathrm{~cm}^{-1}$ are attributed to $\beta$ phase. ${ }^{9}$ They correspond to $\mathrm{CH}_{2}$ rocking $/ \mathrm{CF}_{2}$ asymmetrical stretching and $\mathrm{C}-\mathrm{F}$ stretching vibrations, respectively.

Here, in our ATR-FTIR data, both M-ES and S-ES fibres showed the above three peaks corresponding to $\alpha$ phase, as well as the two peaks for $\beta$ phase. However, for M-ES fibres, the $\beta$ phase peaks were smaller comparing with the case of S-ES. For S-ES fibres, $\alpha$ phase peaks at $761 \mathrm{~cm}^{-1}$ and $976 \mathrm{~cm}^{-1}$ were very small. These results indicates that M-ES samples were $\alpha$ phase rich, while S-ES samples were $\beta$ phase rich, and are consistent with XRD results (Fig. 7).

From these spectra, we evaluated the fraction of $\beta$ phase crystal, $F(\beta)$ by using the following equation:

$$
F(\beta)=\frac{A_{\beta}}{\left(K_{\beta} / K_{\alpha}\right) A_{\alpha}+A_{\beta}}
$$

where $A_{\alpha}$ and $A_{\beta}$ are the absorbances at $761 \mathrm{~cm}^{-1}$ and $840 \mathrm{~cm}^{-1}$, respectively. $K_{\alpha}$ and $K_{\beta}$ indicate the absorption coefficient at the respective wave numbers, which are $6.1 \times 10^{4} \mathrm{~cm}^{2} \mathrm{~mol}^{-1}$ and $7.7 \times 10^{4} \mathrm{~cm}^{2} \mathrm{~mol}^{-1}$, respectively. ${ }^{9,20}$ The obtained $F(\beta)$ values are summarised in Fig. 9 as a function of the rotating speed. The $F(\beta)$ values of the S-ES fibres were higher than those of the M-ES fibres for all rotating speeds, indicating that S-ES is more appropriate for obtaining $\beta$ phase-rich fibres. Considering some references where the finer fibres showed higher $F(\beta)$ values, ${ }^{\mathbf{9}, 21}$ our results also may relate to the difference in the fibre diameters between M-ES and S-ES. According to the explanation by 


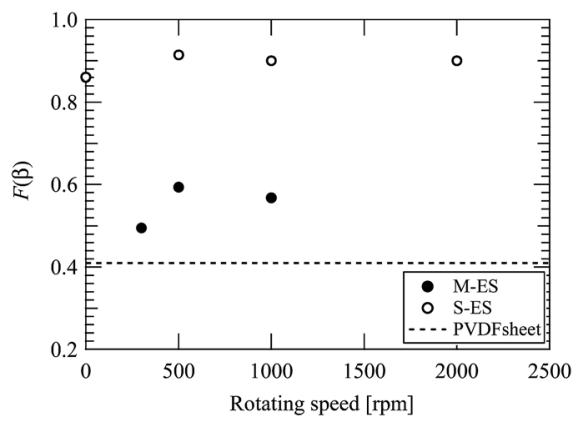

Fig. 9 Variation of $F(\beta)$ values for the fibres spun by M-ES and S-ES, as a function of the rotating speed. The applied voltage was fixed at -10 $\mathrm{kV}$.

Andrew et al. ${ }^{21}$ for the lower viscosity spinning solution, which produces finer fibres, the polymer can be elongated more easily during ES process, resulting in the formation of $\beta$ phase. Another suggested factor is temperature: ${ }^{21}$ Above $90{ }^{\circ} \mathrm{C}$, the polymer chains can slide without changing their conformation. However, below $90{ }^{\circ} \mathrm{C}$, the polymer is stiffer than at higher temperature, and is able to be stretched into $\beta$ phase. In the case of our study, much higher viscosity and temperature during M-ES process must have interfered the formation of $\beta$ phase. In addition, comparing S-ES fibres with the PVDF sheet, S-ES showed larger $F(\beta)$ values. This result is also consistent with that reported by Wu et al. ${ }^{22}$ indicating that the S-ES process induces $\beta$ phase.

Furthermore, we performed DSC measurements and evaluated crystallinity $\left(\chi_{\mathrm{c}}\right)$ from $\chi_{\mathrm{c}}[\%]=\Delta H / \Delta H^{*} \times 100$, where $\Delta H$ and $\Delta H^{*}$ means the heat of fusion obtained from DSC measurement and that for perfect crystal, respectively. We used 104.7 $\mathrm{J} \mathrm{g}^{-1}$ for $\Delta H^{*}$ value. ${ }^{23}$ Fig. 10(a) shows the DSC thermograms for M-ES and S-ES fibres during heating process. The evaluated $\chi_{c}$ values are summarized in Fig. 10(b). It was found that the $\chi_{\mathrm{c}}$ value was about $40 \%$, irrespective of the spinning methods and the applied voltage for M-ES, as well as the rotating speed for S-ES. Here, Gheibi et al. reported the crystallinity was $65 \%$ in the case of S-ES nanofiber. ${ }^{23}$ The lower $\chi_{\mathrm{c}}$ values of our S-ES fibres might be due to the rather short nozzleto-collector distance (i.e. $3.5 \mathrm{~cm}$ ) used in this study. We chose this short distance to match the experimental condition with MES.

As shown in Fig. 10(a), the M-ES fibres showed large melting peak at $169^{\circ} \mathrm{C}$ and small one around $175^{\circ} \mathrm{C}$. On the other hand, the S-ES fibres showed relatively broad single peak around $166^{\circ} \mathrm{C}$. The PVDF sheet showed a single peak at $170.5^{\circ} \mathrm{C}$. Here, as for the assignments of the peaks, there are contradictory references: some researchers mentioned that the peak at higher temperature corresponds to $\beta$ phase, ${ }^{14,21,24}$ while the others described it indicates $\alpha$ phase. ${ }^{22,23,25}$ However, considering our XRD and FT-IR data, we assigned the larger peak of M-ES as the $\alpha$ phase, and the broad peak of S-ES as $\beta$ phase..$^{22,23,25}$ The small peak of M-ES fibres was assigned as $\gamma$ phase. ${ }^{22}$

From these data, we conjectured that $\alpha$ phase was dominant in the PVDF sheet. The M-ES fibre might contain not only $\alpha$ and
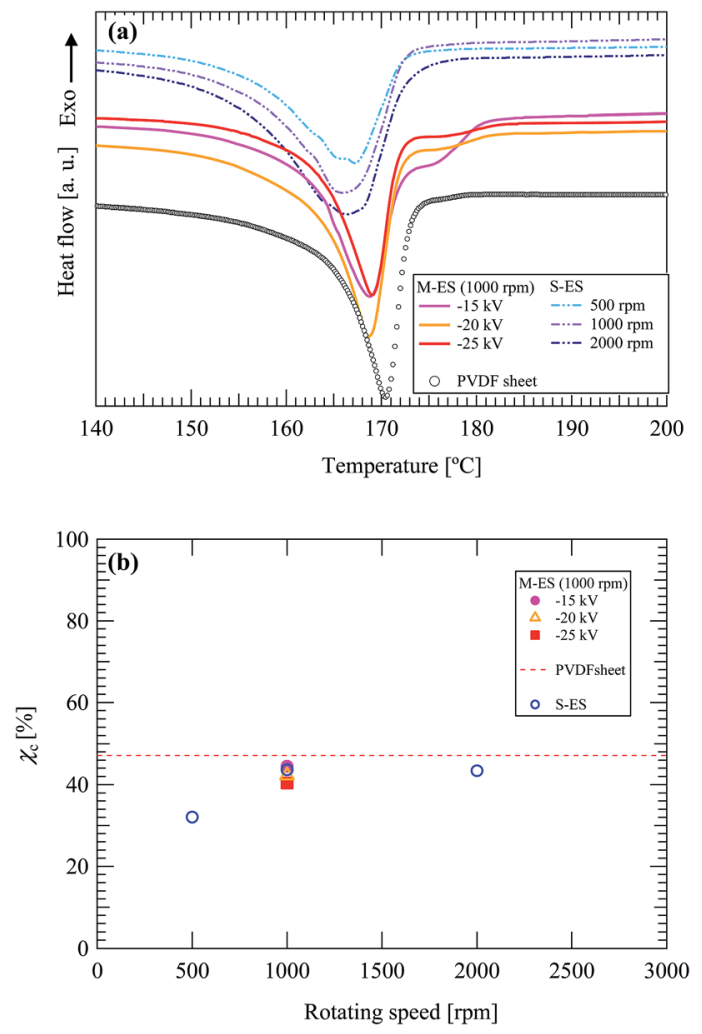

Fig. 10 (a) DSC thermograms for M-ES spun at $1000 \mathrm{rpm}$ with various applied voltages, S-ES fibres spun at various rotating speeds and PVDF sheet used for M-ES. (b) Crystallinity $\left(\chi_{c}\right)$ evaluated from the DSC results.

$\beta$ phases, but also $\gamma$ phase, which is an intermediate phase between $\alpha$ and $\beta$ phases. ${ }^{19}$ The mixture of $\beta$ phase in the M-ES fibres might cause the $\alpha$ phase peak to shift to lower temperature than that in PVDF sheet. However, the existence of $\gamma$ phase in the M-ES fibre could not be confirmed in the ATR-FTIR data, where two peaks should be observed at 812 and $882 \mathrm{~cm}^{-1} .{ }^{21}$ This might be because the amount of $\gamma$ phase was too small to detect by ATR-FTIR. For the S-ES fibres, the broad $\beta$ phase peak might come from the existence of $\alpha$ phase.

From the XRD, ATR-FTIR, and DSC measurements, it was found that the crystallinity of the M-ES fibres were comparable with those obtained by S-ES, but the S-ES fibres had much more $\beta$ phase, which induces piezoelectricity.

\subsection{Effect of spinning conditions on the piezoelectric properties}

Finally, we compared the $d_{33}$ values of the fibres prepared by MES and S-ES. Fig. 11 shows the evaluated $d_{33}$ values as a function of the rotating collector speed. The S-ES fibre mat shows higher $d_{33}$ values, corresponding to the results for the $F(\beta)$. The obtained $d_{33}$ values were much smaller, comparing with those of a bulk PVDF film, ${ }^{26}$ a PVDF nanowire, ${ }^{27}$ or a fibre. ${ }^{28}$ This might be because the porosity of the sample was too high for the measurement system used in this study, considering that the $d_{33}$ values of PVDF sheet measured in this study was $0.30 \pm 0.19$ $\mathrm{pC} / \mathrm{N}$. 


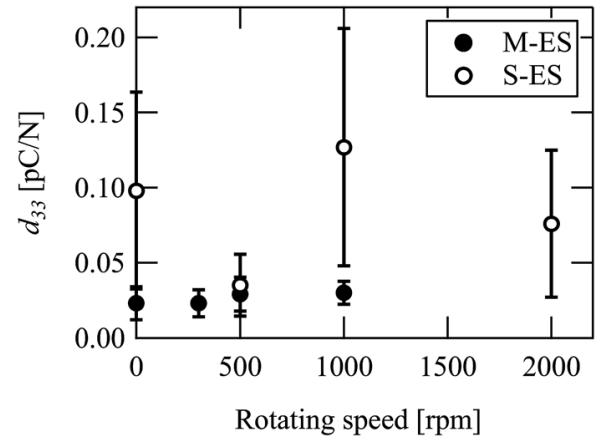

Fig. 11 Variation of $d_{33}$ values for the fibre mats obtained by M-ES and $\mathrm{S}-\mathrm{ES}$ as a function of rotating speed. The applied voltage was $-10 \mathrm{kV}$.

From these experiments, it was found that in the case of $\mathbf{M}$ ES, after-treatment such as drawing may be necessary to obtain $\beta$ phase rich fibres. On the other hand, for the S-ES case, the longer nozzle-to-collector distance, which can elongates the polymer chains more, may enhance the crystallization and the formation of $\beta$ phase.

\section{Conclusions}

We studied the influence of the spinning conditions on the structure and piezoelectricity of the PVDF fibres spun by a linear laser-melt electrospinning process, and compared the results with the corresponding conventional solution-electrospinning process. The rotating speed of a collector and applied voltage only affected the fibre structure and did not strongly influence the crystal structure of the fibres obtained by M-ES. XRD and ATR-FTIR measurements revealed that crystal structure of M-ES fibres was mainly $\alpha$ phase, whereas the S-ES fibres showed higher $\beta$ phase fraction. From DSC measurements, it was found that the crystallinity of M-ES fibres was comparable with that of S-ES.

\section{References}

1 G. M. Sessler, J. Acoust. Soc. Am., 1981, 70, 1596.

2 S. B. Lang and S. Muensit, Appl. Phys. A, 2006, 85, 125-134.

3 K. Tashiro, H. Tadokoro and M. Kobayashi, Ferroelectrics, 1981, 32, 167-175.

4 C. Chang, V. H. Tran, J. Wang, Y. K. Fuh and L. Lin, Nano Lett., 2010, 10, 726-731.

5 Z. H. Liu, C. T. Pan, C. K. Yen, L. W. Lin, J. C. Huang and C. A. Ke, Appl. Surf. Sci., 2015, 346, 291-301.
6 D. Mandal, S. Yoon and K. J. Kim, Macromol. Rapid Commun., 2011, 32, 831-837.

7 T. Lei, L. Yu, G. Zheng, L. Wang, D. Wu and D. Sun, J. Mater. Sci., 2015, 50, 4342-4347.

8 T. Huang, C. Wang, H. Yu, H. Wang, Q. Zhang and M. Zhu, Nano Energy, 2015, 14, 226-235.

9 H. Shao, J. Fang, H. Wang and T. Lin, RSC Adv., 2015, 5, 14345-14350.

10 C. Lee and J. A. Tarbutton, Smart Mater. Struct., 2014, 23, 095044.

11 R. L. Hadimani, D. V. Bayramol, N. Sion, T. Shah, L. Qian, S. Shi and E. Siores, Smart Mater. Struct., 2013, 22, 075017.

12 T. Hattori, M. Kanaoka and H. Ohigashi, J. Appl. Phys., 1996, 79, 2016-2022.

13 A. Hartono, S. Satira, M. Djamal, Ramli and E. Sanjaya, AIP Conf. Proc., 2015, 1656, 030018.

14 A. Salimi and A. A. Yousefi, Polym. Test., 2003, 22, 699-704.

15 E. Nilsson, A. Lund, C. Jonasson, C. Johansson and B. Hagstrom, Sens. Actuators, A, 2013, 201, 477-486.

16 N. Ogata, S. Yamaguchi, N. Shimada, G. Lu, T. Iwata, K. Nakane and T. Ogihara, J. Appl. Polym. Sci., 2007, 104, 1640-1645.

17 N. Shimada, H. Tsutsumi, K. Nakane, T. Ogihara and N. Ogata, J. Appl. Polym. Sci., 2010, 116, 2998-3004.

18 Y. Zheng, L. Cheng, M. Yuan, Z. Wang, L. Zhang, Y. Qin and T. Jing, Nanoscale, 2014, 6, 7842-7846.

19 K. Jurczuk, A. Galeski, M. Mackey, A. Hiltner and E. Baer, Colloid Polym. Sci., 2015, 293, 1289-1297.

20 J. R. Gregorio and M. Cestari, J. Polym. Sci., Part B: Polym. Phys., 1994, 32, 859-870.

21 J. S. Andrew and D. R. Clarke, Langmuir, 2008, 24, 670-672.

22 C. M. Wu and M. H. Chou, Compos. Sci. Technol., 2016, 127, 127-133.

23 A. Gheibi, M. Latifi, A. A. Merati and R. Bagherzadeh, J. Polym. Res., 2014, 21, 469.

24 M. Wu, H. X. Huang and J. Tong, Mater. Des., 2016, 108, 761768.

25 S. S. Choi, Y. S. Lee, C. W. Joo, S. G. Lee, J. K. Park and K. S. Han, Electrochim. Acta, 2004, 50, 339-343.

26 Y. Ting, Suprapto, A. Nugraha, C.-W. Chiu and H. Gunawan, Sens. Actuators, A, 2016, 250, 129-137.

27 V. Cauda, S. Stassi, K. Bejtka and G. Canavese, ACS Appl. Mater. Interfaces, 2013, 5, 6430-6437.

28 J. Pu, X. Yan, Y. Jiang, C. Chang and L. Lin, Sens. Actuators, A, 2010, 164, 131-136. 